\title{
EVALUATION DES SEQUELLES CHEZ LES VICTIMES D'ACCIDENTS DE LA CIRCULATION
}

\author{
T. MASMOUDI, M. BELCADHI*, M. ZEMNI \\ SERVICE DE MEDECINE LEGALE, HÔPITAL UNIVERSITAIRE FARHAT HACHED. SOUSSE \\ *SERVICE D'ORL, HÔPITAL UNIVERSITAIRE FARHAT HACHED. SOUSSE
}

\begin{abstract}
RESUME
La loi n²005-86 du 15 août 2005 a défini les préjudices indemnisables suite à un accident de la circulation: les frais de soins imputables à l'accident, la perte de revenus durant la période d'incapacité temporaire de travail, l'incapacité permanente partielle, le préjudice moral et esthétique, les frais d'assistance d'une tierce personne, le préjudice économique, le préjudice moral et les frais funéraires en cas de décès.

Nous proposons une méthodologie d'évaluation de ces différents chefs de préjudice notamment les incapacités en otorhino-laryngologie.
\end{abstract}

Mots clés : Expertise, Dommage corporel.

\section{SUMMARY}

The law n`2005-86 of 15 August 2005, defined prejudices to indemnify after a road accident: care expenses due to the accident, loss of incomes during the period of temporary inability of work, partial permanent inability, moral and aesthetic prejudice, expenses of assistance by a third person, economic prejudice, moral prejudice and funeral expenses in case of death.

We propose a methodology of assessment of these different chiefs of prejudice, notably in otorhinolaryngology inabilities. Key words: Expertise, Bodily damage.

\section{INTRODUCTION}

Le droit tunisien prévoit qu'un dommage causé à autrui doit être réparé par celui qui en est l'auteur. II est donc nécessaire d'en apprécier la nature et l'importance pour envisager sa réparation. Toute réparation du dommage corporel implique nécessairement une évaluation médicale.

Les préjudices indemnisables dont l'évaluation est du ressort de l'expert sont :

- les frais de soins imputables à l'accident;

- la perte de revenus durant la période d'incapacité temporaire de travail;

- le préjudice corporel : incapacité permanente partielle;

- le préjudice moral et esthétique;

- les frais d'assistance d'une tierce personne;

\section{LA PERIODE D'INCAPACITE TEM- PORAIRE DE TRAVAIL}

L'indemnisation des préjudices suite à l'incapacité temporaire de travail, comporte la perte effective de revenus durant la période d'incapacité fixée dans le certificat médical initial et les certificats médicaux ultérieurs.
Le médecin expert doit alors préciser la période durant laquelle l'arrêt de travail est justifié à temps complet (c'est l'incapacité temporaire totale de travail) ou à temps partiel (c'est l'incapacité temporaire partielle de travail), et dans ce dernier cas il faudra en préciser la hauteur : quart de temps, mi-temps, trois quarts de temps.

Pour les victimes qui n'ont pas d'activités rémunérées (femme au foyer, enfant scolarisé, retraité, etc.), l'incapacité temporaire correspond à la période durant laquelle le patient est totalement dépendant, plus précisément, la durée d'hospitalisation ou d'alitement mais aussi la période pendant laquelle il a besoin de l'aide d'une tierce personne pour effectuer les gestes de la vie quotidienne : s'habiller, se laver, se nourrir, se déplacer, faire sa toilette intime, etc. Cette incapacité temporaire chez un patient non actif ne donnera pas droit à une indemnisation de la perte de revenus, mais permettra d'évaluer d'autres préjudices s'intégrant dans le préjudice moral et esthétique tel que le préjudice douloureux ou les souffrances endurées et permettra de prendre en charge les frais des soins imputables à l'accident.

Dans l'appréciation de la durée de l'incapacité tem- 
poraire, il ne s'agit pas pour le médecin expert d'entériner systématiquement l'arrêt d'activité prescrit par le médecin traitant du patient, mais de déterminer la durée normale de cette interruption compte tenu d'une part de la nature des lésions et de leur évolution et d'autre part du type d'activité de l'intéressé.

\section{LA DATE DE CONSOLIDATION}

La détermination des préjudices définitifs (incapacité permanente partielle, préjudice moral et esthétique, préjudice professionnel) ne peut se faire qu'après la consolidation des lésions.

La consolidation peut être définie comme le moment ou les lésions ne sont plus susceptibles d'évoluer vers l'amélioration ou l'aggravation, sous l'effet d'un traitement quelconque. La guérison est par contre la fin d'évolution des lésions sans séquelles.

Avec la loi du 15 août 2005, la date de consolidation prend une importance quasi-juridique. C'est la date à partir de laquelle court le délai durant lequel l'assurance doit faire l'offre définitive d'indemnisation. Cette dernière doit être présentée dans un délai de deux mois à compter de la date à laquelle l'assureur a eu connaissance de la consolidation des blessures ou de la guérison totale.

La date consolidation est également un repère important en cas d'aggravation des préjudices consécutifs à l'incapacité permanente. En effet aucune réclamation pour l'indemnisation de l'aggravation des préjudices, ne peut être adressée à l'assureur, après l'expiration d'un délai de cinq ans à compter de la date de fixation du taux de l'incapacité définitive.

Compte tenu du délai limité à cinq ans pour reconnaître une aggravation, l'expert peut être amené à repousser la fixation de la date de consolidation pour surseoir à l'appréciation définitive de certaines séquelles pouvant s'aggraver ultérieurement (exemples : sténose laryngo-trachéale, fracture du condyle mandibulaire chez l'enfant, décollement épiphysaire chez l'enfant).

Par ailleurs, après consolidation, certains traitements peuvent être poursuivis uniquement pour stabiliser ou maintenir l'état de santé du patient (et non l'améliorer). Exemple : soins de nursing et de rééducation chez un malade grabataire. Ces traitements représentent des frais futurs qui sont indemnisables sous le chapitre des frais de soins imputables à l'accident.

\section{INCAPACITE PERMANENTE PAR- TIELLE 1- DONNÉES GÉNÉRALES}

La réflexion médico-légale a construit une méthodologie de l'évaluation de l'incapacité en se référant à des barèmes visant à rendre cette appréciation plus précise et à faciliter la compréhension de ce concept par les régleurs et les magistrats.

L'article 131 de loi du 15 août 2005 a défini l'incapacité permanente comme "la réduction définitive de la capacité fonctionnelle de la victime après consolidation exprimée par rapport à sa capacité fonctionnelle juste avant la survenance de l'accident » (1).

L'incapacité correspond ainsi à la diminution des capacités physiques et/ou psychiques d'une victime. Cette diminution est exprimée en taux d'incapacité qui s'étend sur une échelle de 1 à 100\%. Le taux d'incapacité permanente est déterminé par une expertise médicale compte tenu d'un barème officiel. L'évaluation de l'incapacité permanente repose sur une démarche médico-légale que l'on peut résumer en trois étapes

- La première étape est le recueil de l'information à partir de l'interrogatoire du patient, de l'analyse du dossier médical, des différents certificats médicaux et des observations, de l'examen clinique ;

- La seconde étape est le traitement de l'information pour construire un diagnostic positif et définir un processus physiopathologique constituant la base de toute discussion d'imputabilité ;

- La troisième étape est l'exploitation de l'information, c'est-à-dire l'approche fonctionnelle des lésions et déficiences considérées comme imputables à l'accident de la circulation.

L'évaluation de l'incapacité permanente impose également d'étudier la situation antérieure du blessé, en particulier ses antécédents pathologiques et son autonomie dans la vie personnelle et professionnelle par rapport à sa capacité fonctionnelle juste avant la survenue de l'accident.

\section{2 - EVALUATION BARÉMIQUE DES SÉQUELLES ORL}

L'expert $O R L$ doit recourir au nouveau barème paru en 2007 au Journal officiel de la République Tunisienne afin d'évaluer les séquelles consécutives aux accidents de la circulation (2). Ces lignes directrices sont exposées ci-dessous.

\section{a- DOMMAGE AUDITIF Déficit auditif}

Sa détermination repose sur un bilan clinique com- 
plet et sur un bilan para-clinique qui doit comporter au minimum, une impédancemétrie complète (tympanométrie avec recherche du seuil des réflexes stapédiens), une audiométrie tonale subjective liminaire et une audiométrie vocale. Si besoin, la réalité du déficit auditif pourra être confirmée par des tests objectifs (oto-émissions acoustiques, potentiels évoqués auditifs précoces).

Les hypoacousies post-traumatiques ne sont plus évolutives au-delà de 12 mois.

L'évaluation se fait en deux temps:

- Evaluation de la perte auditive moyenne (PAM) par rapport au déficit tonal en conduction aérienne mesuré en décibels sur les fréquences 500, 1000, 2000 et $4000 \mathrm{~Hz}$, en affectant des coefficients de pondération de $2,4,3$ et 1 . La somme est divisée par 10.On se reporte au tableau I, à double entrée, pour l'appréciation des taux.

- Confrontation de ce taux brut aux résultats d'une audiométrie vocale pour apprécier d'éventuelles distorsions auditives (recrutement en particulier) qui aggravent la gêne fonctionnelle. Le tableau II propose les taux de majoration qui peuvent éventuellement être discutés par rapport aux résultats de l'audiométrie tonale liminaire.

L'expert précise si un appareillage est possible. Si la victime est appareillée, l'expert décrit l'amélioration fonctionnelle obtenue, laquelle peut réduire le taux d'incapacité d'au moins 25\%.

\section{Lésions tympaniques}

- Une perforation tympanique sèche n'entraîne pas par elle-même d'incapacité sauf incidence sur l'audition;

- Une otorrhée persistante. .2 à $4 \%$

Sténose du conduit auditif externe. .1 à $3 \%$

\begin{tabular}{|c|c|c|c|c|c|c|c|c|}
\hline $\begin{array}{c}\text { PAM } \\
\text { en dB }\end{array}$ & $\mathbf{0 - 1 9}$ & $\mathbf{2 0 - 2 9}$ & $\mathbf{3 0 - 3 9}$ & $\mathbf{4 0 - 4 9}$ & $\mathbf{5 0 - 5 9}$ & $\mathbf{6 0 - 6 9}$ & $\mathbf{7 0 - 7 9}$ & $\mathbf{8 0}$ et + \\
\hline $\mathbf{0 - 1 9}$ & 0 & 2 & 4 & 6 & 8 & 10 & 12 & $\mathbf{1 4}$ \\
\hline $\mathbf{2 0 - 2 9}$ & 2 & 4 & 6 & 8 & 10 & 12 & 14 & 18 \\
\hline $\mathbf{3 0 - 3 9}$ & 4 & 6 & 8 & 10 & 12 & 15 & 20 & 25 \\
\hline $\mathbf{4 0 - 4 9}$ & 6 & 8 & 10 & 12 & 15 & 20 & 25 & 30 \\
\hline $\mathbf{5 0 - 5 9}$ & 8 & 10 & 12 & 15 & 20 & 25 & 30 & 35 \\
\hline $\mathbf{6 0 - 6 9}$ & 10 & 12 & 15 & 20 & 25 & 30 & 40 & 45 \\
\hline $\mathbf{7 0 - 7 9}$ & 12 & 14 & 20 & 25 & 30 & 40 & 50 & 55 \\
\hline $\mathbf{8 0}$ et + & 14 & 18 & 25 & 30 & 35 & 45 & 55 & $\mathbf{6 0}$ \\
\hline
\end{tabular}

Tableau I : Taux d'incapacité permanente partielle en fonction de la perte auditive

\begin{tabular}{|c|c|c|c|c|c|c|}
\hline $\begin{array}{c}\% \\
\text { Discrimination }\end{array}$ & $\mathbf{1 0 0} \%$ & $\mathbf{9 0 \%}$ & $\mathbf{8 0} \%$ & $\mathbf{7 0} \%$ & $\mathbf{6 0} \%$ & $<\mathbf{5 0 \%}$ \\
\hline $\mathbf{1 0 0} \%$ & 0 & 0 & 1 & 2 & 3 & 4 \\
\hline $\mathbf{9 0 \%}$ & 0 & 0 & 1 & 2 & 3 & 4 \\
\hline $\mathbf{8 0} \%$ & 1 & 1 & 2 & 3 & 4 & 5 \\
\hline $\mathbf{7 0} \%$ & 2 & 2 & 3 & 4 & 5 & 6 \\
\hline $\mathbf{6 0 \%}$ & 3 & 3 & 4 & 5 & 6 & 7 \\
\hline$<\mathbf{5 0} \%$ & 4 & 4 & 5 & 6 & 7 & 8 \\
\hline
\end{tabular}

Tableau II : Majoration des taux d'IPP en fonction du pourcentage de discrimination

\section{Acouphènes et hyperacousies douloureuses}

Leur intensité ressentie n'est pas dépendante de l'importance du déficit auditif. Aucun test ne permet d'objectiver ce trouble. L'expert pourra cependant recourir à une acouphénométrie subjective et à des tests reconnus : questionnaires «DET » et «SEV ». Dans la plupart des cas, il se produit en 12 à 18 mois un phénomène d'habituation cérébrale. On peut alors proposer un taux allant jusqu'à $3 \%$. Lorsque le retentissement psychoaffectif est sévère, la détermination du taux d'incapacité doit se faire dans un cadre multidisciplinaire.

\section{b- TROUBLES DE L'EQUILIBRATION}

- Vertige paroxystique positionnel bénin ........ jusqu'à 4\% - Atteinte vestibulaire périphérique unilatérale (selon la qualité de la compensation) ...............................3 à $8 \%$ - Atteinte vestibulaire destructive périphérique bilatérale ...............................................10 à $20 \%$ - Atteinte déficitaire otolithique . 3 à $5 \%$

En ce qui concerne le syndrome central authentique et objectivé, ce diagnostic doit impérativement être confirmé dans un cadre multidisciplinaire : otoneuro-ophtalmologique. II ne peut être proposé un taux spécifiquement ORL.

\section{c- DOMMAGE VOCAL}

- Aphonie.........................................25\%

- Dysphonie partielle isolée.................jusqu'à 10\%

Les éventuels troubles associés de la déglutition et de la fonction respiratoire seront évalués séparément.

\section{d- DOMMAGE OLFACTIF}

L'exploration de ce sens ne fait appel actuellement qu'à des tests subjectifs de perception et de reconnaissance d'odeur. Ces explorations doivent être effectuées sur chaque fosse nasale séparée. Un déficit de ce type peut, 
ou non, retentir sur le comportement alimentaire du sujet.

- Anosmie : . $.8 \%$

- Hyposmie : 3 à $5 \%$

L'existence de parosmies peut justifier un taux spécifique supplémentaire de $2 \%$. Le retentissement sur le goût ne s'ajoute pas aux taux proposés ci-dessus.

\section{e- DOMMAGE NASAL}

Gène respiratoire (Seule une rhinomanométrie peut permettre une évaluation plus proche de la réalité).

- Diminution unilatérale de la perméabilité nasale...jusqu'à 3\%

- Diminution bilatérale de la perméabilité nasale....jusqu'à 6\%

Perforation septale persistante. jusqu'à 3\% Sinusite chronique post-traumatique (selon l'uni ou la bilatéralité). .jusqu'à $8 \%$

\section{f- DOMMAGE FACIAL}

Paralysie faciale

- Paralysie faciale unilatérale complète et totale.........20\%

- Paralysie faciale unilatérale incomplète (parésie).....2 à 10\%

- Paralysie faciale unilatérale partielle (facial supérieur, facial inférieur).

2 à $10 \%$

- Paralysie faciale bilatérale. $.40 \%$

Hémispasme facial (Selon l'importance de la contracture et la fréquence des crises spastiques)............................................. à $15 \%$

\section{g- DOMMAGE LARYNGE ET TRACHEAL}

En cas de trachéotomie définitive, de trachéomalacie, de sténose trachéale ou de sténose laryngée, le taux d'incapacité permanente partielle doit être évalué en fonction du retentissement sur la respiration (importance de l'insuffisance respiratoire chronique), la déglutition et la phonation.

- Sténose trachéale bien tolérée, sans limitation fonctionnelle pour la vie courante et avec une surveillance espacée. .jusqu'à $5 \%$

- Sténose trachéale bien tolérée, avec une endoprothèse, justifiant une surveillance régulière, et en fonction du retentissement respiratoire. 5 à $10 \%$

- En cas de retentissement respiratoire sévère, le taux de d'incapacité permanente partielle doit être évalué en fonction de ce retentissement.

\section{h- DOMMAGE GUSTATIF}

Agueusie.

\section{LE PREJUDICE MORAL ET ESTHE- TIQUE}

Ce type de préjudice doit être évalué selon son importance par un qualificatif : nul, très léger, léger, modéré, moyen, assez important, important, très important.

Si le domaine et la méthode d'évaluation du préjudice esthétique sont bien connus, il faudrait par contre préciser la nature du préjudice moral qui était avant la promulgation de la loi du 15 août 2005, du domaine d'appréciation du juge.

Rappelons tout d'abord que le préjudice esthétique correspond aux séquelles disgracieuses (cicatrices, déformations statiques ou dynamiques, amputations, mutilations, etc.) surtout dans les régions découvertes exposées au regard car il correspond à la « souffrance morale due au regard des autres ». II est évalué selon deux critères objectifs : l'aspect et le siège de la disgrâce. On tiendra compte des dimensions, de la couleur, de la nature, de la texture et de l'évolution possible de la disgrâce (3).

En ce qui concerne le préjudice moral, il s'agit selon la loi du 15 août 2005 d'un préjudice d'ordre personnel, il devrait recouvrir de notre point de vue les préjudices suivants (1):

- Le préjudice douloureux ou souffrances endurées;

- Le préjudice d'agrément ;

- Le préjudice juvénile.

Le préjudice douloureux correspond aux douleurs subies entre la date de l'accident et celle de la consolidation. De multiples critères servent à l'évaluer: gravité du traumatisme, type des lésions initiales, nombre et gravité des interventions, durée d'hospitalisation et d'immobilisation, existence de complications, nécessité d'un traitement antalgique, durée de la rééducation utile, durée de repos justifiée $(3,4)$.

Le préjudice d'agrément correspond à l'atteinte aux satisfactions et aux plaisirs de la vie du fait des séquelles, soit dans la pratique de certains sports, soit dans la pratique de certains loisirs, qu'ils soient culturels ou non. L'expert doit indiquer si les séquelles sont de nature ou non à entraîner une gêne dans les activités décrites par le patient.

Le préjudice juvénile correspond au retentissement de l'incapacité sur l'avenir de l'enfant, soit pour accéder à certains métiers, soit pour accomplir certains sports. 


\section{LE PREJUDICE PROFESSIONNEL}

Dans le cadre de la loi du 15 août 2005, le médecin expert est tenu de mentionner dans son rapport l'existence ou non d'un préjudice professionnel et le degré de son incidence sur l'activité professionnelle de la victime (1).

L'évaluation du préjudice professionnel se fait par un qualificatif: 1er degré (effet nul), 2ème degré (effet léger), 3ème degré (effet moyen), 4ème degré (effet assez important), 5ème degré (effet important), 6 ème degré (effet très important).

Pour apprécier le préjudice professionnel, l'expert doit s'intéresser à l'état antérieur du sujet, à sa vie professionnelle : métier exercé et conditions d'exercice avant l'accident, conditions de reprise du travail, modification des conditions du travail, voire reclassement ou licenciement. L'expert doit étudier « les gestes professionnels " c'est-à-dire ceux qui sont possibles à exécuter ou au contraire difficiles et même impossibles à exécuter alors qu'ils sont nécessaires à l'exercice du travail.

L'expert ORL soulignera l'importance du retentissement professionnel de la dysphonie chez les professionnels de la voix : enseignants, chanteurs, etc. Un déficit auditif, même léger, peut devenir particulièrement handicapant dans certaines professions : enseignants, commerçants, etc.

\section{AIDE D'UNE TIERCE PERSONNE}

Le législateur a limité la possibilité de recours à l'assistance à la seule situation où la victime présenterait un taux d'incapacité d'au moins $80 \%$ (1).

En pratique l'expert devra spécifier :

- Ce que peut ou ne peut pas faire la victime (gestes,...), avec un descriptif détaillé du déroulement d'une journée à domicile ;

- La nature de l'aide rendue nécessaire par l'incapacité (médicalisée ou non) ainsi que la durée nécessaire dans la journée.

\section{FRAIS FUTURS}

Ils correspondent aux dépenses pour des soins médicaux ou paramédicaux postérieurs à la consolidation, qui sont médicalement prévisibles et répétitifs en raison de la permanence d'états pathologiques chroniques après consolidation définitive.

Ces frais futurs médicalement prévisibles sont aussi médicalement évaluables (ex : périodicité d'hospitalisations, suivi médical avec examens, analyses biologiques et radiographies périodiques, soins infirmiers, etc.).

Parmi ces frais futurs, il faut compter les prothèses (dents, pavillon de l'oreille...) et les appareillages (Prothèse auditive...) nécessaires pour suppléer la fonction physiologique atteinte, ils doivent être évalués en tenant compte de la fréquence de renouvellement et des frais d'entretien (3).

\section{CONCLUSION}

L'expertise médicale du dommage corporel intéresse un être humain et l'on sait que la médecine est à la fois un art et une science, ceci altère parfois la rigueur que l'on est en droit d'attendre d'une expertise.

A cet égard, il importe de rappeler la réflexion de LE PRINCE RINGUET «En mathématiques, on a toutes les données et il n'y a qu'une seule solution; dans la vie, on n'a pas toutes les données et il y a plusieurs solutions ». C'est dire que pour l'appréciation du dommage corporel à la lumière de la nouvelle loi, des questionnements restent possibles, et il est capital que l'approche de la vérité et de l'exactitude résulte d'une méthodologie rigoureuse qu'il conviendra de parfaire par la concertation entre l'expert et le régleur.

\section{REFERENCES}

1. Loi n²005-86 du 15 aout 2005 , portant insertion d'un cinquième titre au code des assurances, concernant l'assurance de la responsabilité civile du fait de l'usage des véhicules terrestres à moteur et au régime d'indemnisation des préjudices résultant des atteintes aux personnes dans les accidents de la circulation. Imprimerie officielle de la république tunisienne, Journal Officiel de la République Tunisienne, $2005 ; n^{\circ} 67:$ 2182-2191.

2. Barème d'évaluation médico-légale des incapacités permanentes des vic- times d'accidents de la circulation. Imprimerie officielle de la république tunisienne. Journal Officiel de la République Tunisienne, 2007, n49: 2105-2153. 3 . Arbus L., Rougé D., Le rôle du médecin traitant dans les expertises. Paris, Eska, 1995.

4. Hureau J. , Poitout D.. L'expertise médicale en responsabilité médicale et en réparation d'un dommage corporel. Paris, Masson, 2005. 